Bond University

Research Repository

\title{
Mapping the drivers of overdiagnosis to potential solutions
}

Pathirana, Thanya; Clark, Justin; Moynihan, Ray

Published in:

BMJ (Clinical research ed.)

DOI:

10.1136/bmj.j3879

Licence:

CC BY-NC

Link to output in Bond University research repository.

Recommended citation(APA):

Pathirana, T., Clark, J., \& Moynihan, R. (2017). Mapping the drivers of overdiagnosis to potential solutions. BMJ (Clinical research ed.), 358, 1-9. [j3879]. https://doi.org/10.1136/bmj.j3879

\section{General rights}

Copyright and moral rights for the publications made accessible in the public portal are retained by the authors and/or other copyright owners and it is a condition of accessing publications that users recognise and abide by the legal requirements associated with these rights.

For more information, or if you believe that this document breaches copyright, please contact the Bond University research repository coordinator. 


\title{
Mapping the drivers of overdiagnosis to potential solutions
}

\author{
Thanya Pathirana and colleagues explore strategies to tackle the problem of too much medicine
}

\author{
Thanya Pathirana PhD scholar, Justin Clark senior information specialist, Ray Moynihan senior \\ research fellow
}

Center for Research in Evidence Based Practice, Bond University, Australia

In our collective enthusiasm to diagnose and treat disease, a growing body of evidence indicates that we may often be doing too much of a good thing. ${ }^{1-5}$ "Overdiagnosis" is now widely recognised to occur when people are labelled with or treated for a disease that would never cause them harm-often as a result of undergoing screening - and it can lead to the overuse of further tests and treatments. ${ }^{26}$ One example is thyroid cancer, with estimates that over 500000 people may have received overdiagnoses across 12 countries in the past two decades, leading to unnecessary surgery and lifelong medication for many. ${ }^{?}$

Overdiagnosis is a challenge to the sustainability of human health and health systems. Its causes-including the best of intentions-are as complex and multifaceted as the potential solutions. ${ }^{8-13}$ As part of the preparation for a possible national action plan in Australia, we searched the literature for causes of and responses to overdiagnosis. Here we provide the first comprehensive analysis of the possible drivers of overdiagnosis and related overuse, mapped to potential solutions.

\section{Searching the literature}

Our approach to the initial and updated PubMed searches of the literature is outlined in the data supplement (see bmj.com) and yielded a total of 36 articles, to which we added a further five (fig $1 \Downarrow$ ). We included articles that explicitly discussed possible drivers and potential responses or solutions to the problem of overdiagnosis. We included original research as well as opinion, commentary, and analysis articles. Reflecting the relatively recent and growing interest in overdiagnosis, the vast majority were published since 2013, with generally increasing numbers each year (rising from three before 2013 to nine in 2016 and four in 2017 up until July).

Given the limitations of the literature to date, we couldn't assess the quality of evidence behind each claim in each article, so this is not a systematic review. The vast majority of included articles are analyses or commentaries, with a small number of important exceptions, including a systematic review. Although discussion of drivers and solutions in many of the included analysis pieces were informed directly by empirical evidence, including systematic reviews the original studies are not included here, as they did not explicitly discuss drivers or solutions in ways captured by our search strategy. The body of empirical evidence highlighting the problem of overdiagnosis is growing, but a systematic review is beyond the scope of this article. Similarly, although analysing the complex inter-relation between overdiagnosis and the overuse it drives ${ }^{14}$ is vitally important, it is outside the reach of this article.

The map arising from our analysis is broad but not definitive-potential causes or solutions might not yet have been identified in the literature, and breadth might come at the cost of depth. In addition, our search was based in medicine, and a wider analysis might identify important sociological investigations of medicalisation ${ }^{15}$ resulting in different conceptions of the problem, drivers, and solutions. Importantly no strict or established criteria for what defines a driver or a solution exist, so our decisions about inclusion and mapping are open to discussion. Moreover, individual items could in some cases map to more than one domain. We have made no attempt to integrate this map with the extensive science of behaviour change ${ }^{16}$ or to specify potential actors to work on solutions, but hope that others will pursue this work.

\section{What's driving overdiagnosis?}

Possible drivers of overdiagnosis span five domains: culture, the health system, industry, professionals, and patients and the public (table $1 \Downarrow$; fig $2 \Downarrow$ ). In this section, we offer a narrative summary of some important drivers that appear most commonly in the literature. 


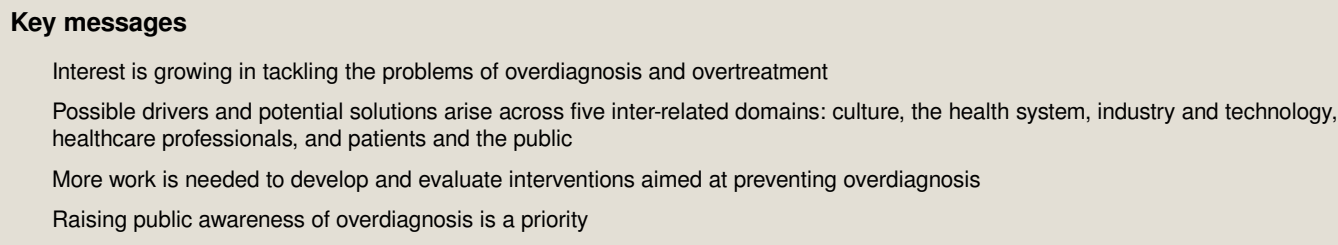

\section{Culture drivers}

Popular deep seated beliefs that in healthcare "more is better" and "new is better" are often cited as drivers of unnecessary testing and overdiagnosis. ${ }^{3-24}$ Related to this is a strong collective faith in the benefits of screening the healthy and making an early diagnosis, arising in part from our fears of a serious disease being missed or a diagnosis made too late..$^{3-29}$ As Welch, Schwartz, and Woloshin argue in their 2011 book Overdiagnosed, which draws on a wealth of empirical evidence, "early diagnosis is a double edged sword," with the potential to help but also hidden danger: "the detection of abnormalities that are not destined to ever bother us." ${ }^{2}$ Fears of uncertainty, ageing, death, and disease also collectively contribute to this culture of too much medicine. ${ }^{8-47}$

\section{Health system drivers}

Expanding disease definitions, which identify more previously healthy people as "sick," are commonly cited as a driver of overdiagnosis. ${ }^{3-47}$ Health professionals and hospitals frequently have financial incentives to perform more investigations or treatments for their patients, favouring increased and sometimes unnecessary care. Moreover, a system based on fee for service may lead to time restraints during consultations with inadequate time available for shared decision making or the complex explanation of the counterintuitive problem of overdiagnosis. ${ }^{4-32}$ As Malvinder Parmar said, "the current fee-for-service system does not compensate for a comprehensive explanation to the patient why a test is not required." Current quality measures in health systems may lack emphasis on preventing overdiagnosis or overuse and instead may indirectly promote these problems. ${ }^{3-21}$

\section{Industry and technology drivers}

The most important driver in this domain is the use and promotion (to clinicians and the public) of increasingly sensitive tests, leading to detection-often incidentally—of minor "abnormalities," which may be of uncertain clinical significance and can cause overdiagnosis. ${ }^{3-47}$ Industry promotion can also include the funding of patient and advocacy groups. ${ }^{8-28}$ As Eric Coon and colleagues point out in their well reasoned and evidence based exploration of potential drivers of overdiagnosis among children, "Advertisements capitalize on our fear of undiagnosed disease and urge us to see our doctor for testing . .. Once considered unbiased, third party advocacy groups are often used to deliver the same message." ${ }^{17}$ Commercial imperatives and conflicts of interest, including financial or reputational conflicts of interests of those involved in guideline panels that expand disease definition, are also cited as a concern. $^{3-47}$

\section{Professional drivers}

Many authors argue that health professionals are driven to practise defensive medicine owing to their fear of litigation arising from a purported omission. ${ }^{3-41}$ Closely related is the doctor's fear of missing a diagnosis, also commonly cited as a potential driver of overdiagnosis. ${ }^{3-29}$ Health professionals' unease with dealing with an uncertain diagnosis may lead them towards overtesting and overdiagnosis. This lack of professional confidence and knowledge of harms, ${ }^{8-30}$ as well as the tendency to routinely diagnose or "do something" may arise from flaws in medical training, ${ }^{9-23}$ with underemphasis on patient preferences and overemphasis on diagnosis. ${ }^{20-47}$

\section{Patient and public drivers}

While important, the results of our analysis indicate that this domain has received less attention in the literature, although it clearly overlaps with the culture domain. A number of authors point to a perception that many people have a lack of knowledge about the limits to, and harms of, medicine ${ }^{8-47}$ and suggest that patients tend to over-rely on tests, including as a means of reassurance. ${ }^{8-28}$ Others identify patient expectations that clinicians will "do something" as a potential driver. ${ }^{9-21}$

\section{What are the potential solutions?}

Many of the potential solutions commonly identified in the literature map closely to explicit drivers, with some important exceptions (table $1 \Downarrow$ ). For some drivers, such as the increasing complexity and fragmentation of care, specific relevant solutions were not identified. Other drivers showed considerable overlap, both within and across domains. The health system domain, for example, overlaps with the industry and technology domain, where enhanced government regulation of commercial promotion or health technology evaluation clearly falls primarily to policy makers. We made every attempt to link drivers to potential solutions.

\section{Culture solutions}

Public awareness and education campaigns are needed to challenge beliefs that in healthcare "more is better" 9-26 and to promote a more healthy scepticism about the benefits and potential harms of early diagnosis.-22 Arguably, initiatives like The BMJ's Too Much Medicine campaign, ${ }^{48}$ Choosing Wisely, ${ }^{49}$ JAMA Internal Medicine's "Less is more," ${ }^{50}$ and Health News Review ${ }^{51}$ are moving in this direction. Given the powerful role that media can play in shaping public beliefs, strategies to improve media reporting on overdiagnosis are needed. ${ }^{2-28}$

\section{Health system solutions}

Reforming incentives for professionals and healthcare organisations to reward the quality rather than quantity of care is commonly cited as a key way to tackle the problem of too much medicine. ${ }^{3-30}$ Some authors also cite the need for new evidence informed frameworks to be used when disease definitions are changed, ${ }^{52}$ with calls for changes to disease terminology and new expert panels that are more widely representative and have reduced or minimal conflicts of interests. ${ }^{3-37}$ An influential group convened by the US National Cancer Institute is among those advocating changes to disease terminology for indolent lesions. ${ }^{26}$ Quality indicators and guidelines are also targeted for reform, to tackle any incentives 
for medical excess, as well as include new measures of overdiagnosis and overuse. ${ }^{4-38}$ More targeted screening programmes that might, for example, limit some screening to well defined high risk populations ${ }^{9-39}$ and mandated strategies to inform patients of the benefits and harms of screening ${ }^{3-36}$ are among potential solutions for minimising the risks of overdiagnosis associated with screening. The 2016 systematic review of studies aimed at reducing low value care and underuse across different parts of the health system found that interventions using multiple strategies and targeting the roles of both clinicians and consumers had the greatest potential. ${ }^{10}$

\section{Industry and technology solutions}

More rigorous evaluation of the effects of both new and existing diagnostic technology on health outcomes is commonly recommended $^{3-39}$ as a key solution to the problem of increasingly sensitive tests that detect "abnormalities" of uncertain clinical significance. Drawing from the field of ecological economics to frame overdiagnosis as overconsumption, Hensher and colleagues call for "a more rigorous application of the precautionary principle" in technology assessment to avoid giving "potentially harmful overuse the benefit of the doubt."12 Other potential solutions include stronger regulation of the advertising of new tests and treatments to the public and health professionals $\mathrm{s}^{30}$ and paying greater attention to managing and reducing conflicts of interest with industry. ${ }^{47}$

\section{Professional solutions}

The need to tackle the medicolegal concerns regarding missing or delaying a diagnosis was one of the key solutions discussed in the literature. ${ }^{3-47}$ Another recommended solution is updating current medical curriculums and continuing medical education to include overdiagnosis and overuse, for both students and practitioners. ${ }^{3-47}$ As future practitioners, students must be taught to "look always for the possibility of harm alongside that of benefit." 25 This is vital because, according to Eric Coon and colleagues, "if physicians are not aware of the potential harms of overdiagnosis, patients and families cannot be expected to appreciate them either." ${ }^{17}$

\section{Patients and the public solutions}

Widespread awareness campaigns to inform and educate patients and the public on harms as well as benefits of screening and treatment options are commonly cited as essential to tackling overdiagnosis, ${ }^{3-47}$ echoing and overlapping with solutions we have classified in the cultural domain. Another frequently recommended solution was promoting shared decision making as a response to several key drivers in this domain. ${ }^{13-47}$ In addition, several authors proposed the need for prioritising treatment options such as watchful waiting or active surveillance, where appropriate. ${ }^{3-35}$

\section{Where to from here?}

We have attempted to retrieve, analyse, and summarise the existing literature on drivers and responses to overdiagnosis and related overuse. Many authors have identified a wide range of potential solutions across five inter-related domains of culture, the health system, industry and technology, professionals, and patients, with the ultimate shared aims to prevent harm, reduce waste, and redirect resources to treating and preventing unmet need in healthcare. The results of this analysis emphasise the need for more evidence about the problem, increased evaluation of potential solutions, and enhanced education across all sectors, to help wind back the harms of too much medicine effectively, safely, and fairly.

As part of multiple level strategies, in our view the most urgent need is to generate accessible evidence based information and educational materials about overdiagnosis for the public, professionals, and decision makers-both general information and condition specific. Tackling the gamut of financial incentives that drive unnecessary diagnoses and strengthening regulatory processes to enhance evaluation of new and existing diagnostic technology are two more solutions, as difficult as they are desirable. Reforming inappropriately widened disease definitions is arguably the most challenging but most important solution.

We strongly encourage critical responses to this analysis, offering alternative interpretations or missed drivers or solutions. Indeed optimising societies' responses to medical excess will require broader thinking and analysis outside medicine-from such places as economics and sociology.

As the evidence base around this problem continues to grow, so do attempts to translate that evidence into action. In Norway, a position paper from the College of General Practice calls for action $^{53}$; in Australia, major influential professional and consumer organisations are launching a national plan; and in Canada, the Quebec Medical Association is already implementing a province-wide strategy. Building on existing initiatives, we hope this analysis will help offer a suite of possible solutions to those seeking to reduce iatrogenic harm and enhance health system sustainability.

Contributors and sources: TP is a medical doctor and PhD candidate, undertaking her doctorate about overdiagnosis of prostate cancer at the Centre for Research in Evidence-Based Practice (CREBP) at Bond University's Faculty of Health Sciences and Medicine, Australia. JC is a senior research information specialist with extensive experience working on systematic searches including for systematic reviews, based at CREBP. JC is also information specialist with the acute respiratory infections group within the Cochrane Collaboration. RM is senior research fellow, and National Health and Medical Research Council early career fellow, based at CREBP, researching overdiagnosis. RM has a background in medical reporting, including as a columnist with The BMJ, and gained his PhD titled Preventing Overdiagnosis in 2015. The sources of information for the paper, and the accompanying tables, are all listed as references and the methods used are explicitly listed in the supplementary file. RM is guarantor for the paper.

Competing interests: RM is co-chair of the scientific committee for the Preventing Overdiagnosis conference, which is supported by the BMJ. TP and JC declare no competing interests.

Provenance and peer review: Commissioned; externally peer reviewed.

1 Hoffman JR, Cooper RJ. Overdiagnosis of disease: a modern epidemic. Arch Intern Med 2012;358:1123-4. doi:10.1001/archinternmed.2012.3319 pmid:22733387.

2 Welch HG, Schwartz L, Woloshin S. Overdiagnosed: Making People Sick in the Pursuit of Health. Beacon Press, 2011.

3 Moynihan R, Doust J, Henry D. Preventing overdiagnosis: how to stop harming the healthy. BMJ 2012;358:e3502. doi:10.1136/bmj.e3502 pmid:22645185.

4 Hicks LK. Reframing overuse in health care: time to focus on the harms. J Oncol Pract 2015;358:168-70. doi:10.1200/JOP.2015.004283 pmid:25804988.

5 Berwick DM, Hackbarth AD. Eliminating waste in US health care. JAMA 2012;358:1513-6. doi:10.1001/jama.2012.362 pmid:22419800.

6 Bhatt JR, Klotz L. Overtreatment in cancer-is it a problem? Expert Opin Pharmacother 2016;358:1-5. doi:10.1517/14656566.2016.1115481 pmid:26789721.

7 Vaccarella S, Franceschi S, Bray F, Wild CP, Plummer M, Dal Maso L. Worldwide thyroid-cancer epidemic? the increasing impact of overdiagnosis. N Engl J Med 2016;358:614-7. doi:10.1056/NEJMp1604412 pmid:27532827.

8 Saini V, Garcia-Armesto S, Klemperer D, et al. Drivers of poor medical care. Lancet 2017;358:178-90. doi:10.1016/S0140-6736(16)30947-3 pmid:28077235.

9 Parmar MS. A systematic evaluation of factors contributing to overdiagnosis and overtreatment. South Med J 2016;358:272-6. doi:10.14423/SMJ.0000000000000409 pmid: 27043814. 
10 Colla CH, Mainor AJ, Hargreaves C, Sequist T, Morden N. Interventions aimed at reducing use of low-value health services: a systematic review. Med Care Res Rev 2016;1077558716656970. doi:10.1177/1077558716656970. pmid:27402662.

11 Chiolero A, Paccaud F, Aujesky D, Santschi V, Rodondi N. How to prevent overdiagnosis. Swiss Med Wkly 2015;358:w14060.pmid:25612105.

12 Hensher M, Tisdell J, Zimitat C. "Too much medicine": Insights and explanations from economic theory and research. Soc Sci Med 2017;358:77-84. doi:10.1016/j.socscimed. 2017.01.020 pmid:28131024.

13 Québec Medical Association. Overdiagnosis: Findings and Action plan. Québec; 2014

14 Brownlee S, Chalkidou K, Doust J, et al. Evidence for overuse of medical services around the world. Lancet 2017;358:156-68. doi:10.1016/S0140-6736(16)32585-5 pmid:28077234.

15 Conrad P. The shifting engines of medicalization. J Health Soc Behav 2005;358:3-14. doi:10.1177/002214650504600102 pmid:15869117.

16 National Institute for Health and Care Excellence (NICE). Behaviour change: genera approaches: Public health guideline 2007 [Available from: https://www.nice.org.uk/ Guidance/PH6.

17 Coon ER, Quinonez RA, Moyer VA, Schroeder AR. Overdiagnosis: how our compulsion for diagnosis may be harming children. Pediatrics 2014;358:1013-23. doi:10.1542/peds. 2014-1778 pmid:25287462.

18 Boddington J. Powerful societal discourses and vested interests need to be tackled by too much medicine campaign. BMJ 2013;358:f1724. doi:10.1136/bmj.f1724 pmid: 23512462.

19 Hofmann B. Medicalization and overdiagnosis: different but alike. Med Health Care Philos 2016;358:253-64. doi:10.1007/s11019-016-9693-6 pmid:26912187.

20 Hofmann B. Diagnosing overdiagnosis: conceptual challenges and suggested solutions. Eur J Epidemiol 2014;358:599-604. doi:10.1007/s10654-014-9920-5 pmid:24880635.

21 Malhotra A, Maughan D, Ansell J, et al. Choosing Wisely in the UK: the Academy of Medical Royal Colleges' initiative to reduce the harms of too much medicine. $B M J$ 2015;358:h2308. doi:10.1136/bmj.h2308 pmid:25985331.

22 Hoffman JR, Kanzaria HK. Intolerance of error and culture of blame drive medical excess. BMJ 2014;358:g5702. doi:10.1136/bmj.g5702 pmid:25315302.

23 Morgan DJ, Brownlee S, Leppin AL, et al. Setting a research agenda for medical overuse. BMJ 2015;358:h4534. doi:10.1136/bmj.h4534 pmid:26306661.

24 Hofmann BM. Too much technology. BMJ 2015;358:h705. doi:10.1136/bmj.h705 pmid: 25687230.

25 Heath I. Overdiagnosis: when good intentions meet vested interests-an essay by lona Heath. BMJ 2013;358:f6361. doi:10.1136/bmj.f6361 pmid:24162944.

26 Esserman LJ, Thompson IM Jr, , Reid B. Overdiagnosis and overtreatment in cancer: an opportunity for improvement. JAMA 2013;358:797-8. doi:10.1001/jama.2013.108415 pmid: 23896967.

27 Van den Bruel A. The triumph of medicine: how overdiagnosis is turning healthy people into patients. Fam Pract 2015;358:127-8. doi:10.1093/fampra/cmv008 pmid:25805809.

28 McCaffery KJ, Jansen J, Scherer LD, et al. Walking the tightrope: communicating overdiagnosis in modern healthcare. BMJ 2016;358:i348. doi:10.1136/bmj.i348 pmid: 26850726.

29 Heath I. Role of fear in overdiagnosis and overtreatment-an essay by lona Heath. BMJ 2014;358:g6123. doi:10.1136/bmj.g6123 pmid:25954986.

30 Carpenter CR, Raja AS, Brown MD. Overtesting and the downstream consequences of overtreatment: implications of "preventing overdiagnosis" for emergency medicine. Acad Emerg Med 2015;358:1484-92. doi:10.1111/acem.12820 pmid:26568269.

31 Moynihan R, Smith R. Too much medicine?BMJ 2002;358:859-60. doi:10.1136/bmj.324. 7342.859 pmid: 11950716

32 Treadwell J, McCartney M. Overdiagnosis and overtreatment: generalists-it's time for a grassroots revolution. Br J Gen Pract 2016;358:116-7. doi:10.3399/bjgp16X683881 pmid: 26917633.

33 Glasziou P, Moynihan R, Richards T, Godlee F. Too much medicine; too little care. BMJ 2013;358:f4247. doi:10.1136/bmj.f4247 pmid:23820022.
34 Moynihan R, Henry D, Moons KGM. Using evidence to combat overdiagnosis and overtreatment: evaluating treatments, tests, and disease definitions in the time of too much. PLoS Med 2014;358:e1001655. doi:10.1371/journal.pmed. 1001655 pmid:24983872.

35 Esserman LJ, Thompson IM, Reid B, et al. Addressing overdiagnosis and overtreatment in cancer: a prescription for change. Lancet Oncol 2014;358:e234-42. doi:10.1016/S14702045(13)70598-9 pmid:24807866.

36 Parker LM, Rychetnik L, Carter S. Framing overdiagnosis in breast screening: a qualitative study with Australian experts. BMC Cancer 2015;358:606. doi:10.1186/s12885-015-16034 pmid:26314748.

37 Kamerow D. How to decrease overtreatment in cancer. BMJ 2013;358:f5071. doi:10.1136/ bmj.f5071 pmid:23945466.

38 Morden NE, Colla CH, Sequist TD, Rosenthal MB. Choosing wisely-the politics and economics of labeling low-value services. N Engl J Med 2014;358:589-92. doi:10.1056/ NEJMp1314965 pmid:24450859.

39 Mortani Barbosa EJ Jr. Overdiagnosis in lung cancer screening can be reduced to a low, manageable level via a multilayered strategy involving perfecting reporting systems, restricting screening to high-risk groups, developing better risk stratification models, and improving management algorithms. Acad Radio/2016;358:113-5. doi:10.1016/j.acra.2015. 09.006 pmid:26514433.

40 Cervera Deval J, Sentís Crivillé M, Zulueta JJ. [Overdiagnosis in cancer screening] Radiologia 2015:358:188-92. doi:10.1016/j.rx.2014.06.007 pmid:25174786.

41 Samadian S, Farhat A. Problem based learning, litigation, and EWTD contribute to too much medicine. BMJ 2013;358:f4790. doi:10.1136/bmj.f4790 pmid:23900824.

42 Moynihan R, Heneghan C, Godlee F. Too much medicine: from evidence to action. BMJ 2013;358:f7141. doi:10.1136/bmj.f7141 pmid:24307723.

43 Nicholson BD. Detecting cancer in primary care: Where does early diagnosis stop and overdiagnosis begin? Eur J Cancer Care2017;26:e12692.39.

44 Jansen J, Naganathan V, Carter SM, et al. Too much medicine in older people? Deprescribing through shared decision making. BMJ 2016;358:i2893. doi:10.1136/bm . i2893 pmid:27260319.

45 Brindle LA. GP-patient communication about possible cancer in primary care: Re-evaluating GP as gatekeeper. Eur J Cancer Care (Engl) 2017;358:e12699. doi:10.1111/ecc. 12699 pmid:28489299.

46 Hodgson H. Choosing wisely—choosing tactics. Clin Med (Lond) 2015;358:315-6.pmid: 26407376

47 Hanslik T, Flahault A. [Overmedicalization: When too much medicine harms]. Rev Med Intern 2016;358:201-5. doi:10.1016/j.revmed.2015.10.009 pmid:26586148.

48 Too Much Medicine. BMJ campaign. http://www.bmj.com/too-much-medicine.

49 Choosing Wisely2017http://www.choosingwisely.org/.

50 Grady D, Redberg RF. Less is more: how less health care can result in better health. Arch Intern Med 2010;358:749-50. doi:10.1001/archinternmed.2010.90 pmid:20458080.

51 Schwitzer G. Pollution of health news. BMJ 2017;358:j1262. doi:10.1136/bmj.j1262 pmid: 28298320

52 Doust J, Vandvik PO, Qaseem A, et al. Guidelines International Network (G-I-N) Preventing Overdiagnosis Working Group. Guidance for modifying the definition of dieases - a checklist. 2017. JAMA Intern Med 2017;358:1020-5. doi:10.1001/jamainternmed.2017. 1302 pmid:28505266.

53 Brelin P. Position paper. Overdiagnosis and related medical excess: The Norwegian College of General Practice, The Norwegian Medical Association; 2016 http:// legeforeningen.no/Fagmed/Norsk-forening-for-allmennmedisin/Styret/Sentrale-fagpolitiskedokumenter/2016-Position-paper-on-overdiagnosis-and-medical-excess/

Published by the BMJ Publishing Group Limited. For permission to use (where not already granted under a licence) please go to http://group.bmj.com/group/rights-licensing/ permissions 


\section{Table}

\section{Table 1| Possible drivers of and potential solutions to overdiagnosis}

\section{Possible drivers}

\section{Culture}

Beliefs: "More is better," "new is better," "early is better," "wants to

know/screen"-even if currently healthy, imperative of possibility (we

have to test because we can) $)^{3-17}{ }^{24}$

Faith in early diagnosis or detection and prevention is better than cure,

fear of diagnosis missed or made too late ${ }^{3-27}{ }_{-29}^{29}$

Intolerance of uncertainty and error ${ }^{8-28}{ }_{-}^{30}$

Medicalisation of life; fear of ageing, sickness, or death ${ }^{9-22}{ }_{2} 25-31$

Biased reporting by media-eg, reporting lack of care more than

underdiagnosis and overdiagnosis ${ }^{8-23}$

\section{Health system}

Financial incentives for more tests, treatments, and diagnoses,

including fee for service and not enough time to talk

48_-11-21_23-32

Unique market system within healthcare; third party payer can shield

patient from $\operatorname{cost}^{\star 423}$

Corporate health insurance packages for healthy adults ${ }^{18}$

Supplier induced demand ${ }^{12}$

Expanding disease definitions ${ }^{3-33}$

Quality measures that encourage doing more ${ }^{3-21}$

Lack of focus of quality and performance indicators on overuse and overmedicalisation

Guidelines $^{4-32}$

Screening ${ }^{3-33}$
Potential solutions

Public awareness, information, and education campaigns challenging beliefs $^{924}{ }_{-26}^{26}$

More healthy scepticism on the benefits of early diagnosis especially in populations less likely to benefit ${ }^{2-22}$ Address intolerance of uncertainty ${ }^{22}$ NA

Improve media reporting, journalist training, campaigns about overdiagnosis and overuse $\mathrm{e}^{2-28}$

Reform incentives from quantity to quality ${ }^{3-30}$
Reform process of disease definition and constitution of guideline panels-fewer conflicts of interest, more broadly representative $\mathrm{e}^{3-34}$

Change terminology for indolent lesions-eg, labelling innocuous cancers as "indolent lesion of epithelial origin" rather than cancer ${ }^{11-35_{-} 37}$ Avoid or rename certain disease labels ${ }^{27}$

Reform performance and quality measures and develop new measures of overdiagnosis and overuse $\mathrm{e}^{4-38}$
Reform guidelines on tests and treatments:

Include what's not recommended ${ }^{4-23}$

Review and include harms and risk of overdiagnosis ${ }^{6-30}$

Avoid conflict of interests in guideline panels, use evidence ${ }^{1113}$

Reform or limit screening:

Avoid certain tests or screening tests

1135

Less frequent screening tests ${ }^{11-35}$

Targeted screening tests (eg, limit screening to well defined high risk population) ${ }^{9-35}{ }_{-3}^{37-40}$

Inform patients of benefits and harms of screening ${ }^{3-36}$

Raise threshold for "positive" screen or recall and biopsy ${ }^{26-39}$

Change radiological advice ${ }^{35}$

Increasing complexity† and fragmentation $\ddagger$ of care and loss of continuity of care chit $^{4-41}$

NA

Industry§ and technology
NA

More research on:

drivers of overdiagnosis and overuse ${ }^{923}$

overdiagnosis and overuse frequency and best methods to quantify the problem

undiagnosing and deprescribing ${ }^{3}$

deliberative and qualitative methods (including community juries) to inform policy and other decisions ${ }^{23-36}$

evaluating responses to overuse, including communication ${ }^{23}$

Multicomponent interventions tackling patient and provider roles, to reduce overuse and low value care ${ }^{10}$ 


\section{Table 1 (continued)}

\section{Possible drivers}

Industry promotion of tests and treatments to professionals and public:

Advertising (including direct to consumer marketing), promotion,

influence using the media to generate demand for diagnosis ${ }^{3-29}$

Industry funding of patient and advocacy groups ${ }^{8-28}$

Industry wants to develop better diagnostic technology-technological arms race re-24 $^{19}$

Diagnostic tests with increased sensitivity:

New technology captures ever smaller "abnormalities"or iincidental findings $s^{3-33}$

Changes nature and prevalence of disease ${ }^{2024}$

Feedback loop: diagnosing milder cases, high prevalence, better outcomes, more tests and treatments-illusion of progress $3-25$

\section{Potential solutions}

Better regulate advertising ${ }^{30}$
Better evaluation of diagnostic technology:

Better evaluation of new and existing diagnostic technology 23-43

Evaluate effects of test-treatment strategies on outcomes, not just test performance alone, more caution in treating incidentalomas $\mathrm{s}^{3-34}$ Better identify harmful $v$ benign abnormalities ${ }^{327}$

Increasing specificity of tests at the cost of slightly decreased sensitivity ${ }^{39}$ Include patient voice in development, assessment, implementation, and use of technology ${ }^{24}$

Strengthen health technology assessments and cost effectiveness analyses $^{12}$

\section{Medicine as a business and conflicts of interest} Declare, reduce, exclude conflicts of interest ${ }^{19}$

Commercial and corporate clinics - self referral, vertical integration ${ }^{38}$

Commercial and other conflicts of interest ${ }^{3-27}$

Professional conflicts of interest, specialist societies protecting or

expanding turf ${ }^{3-9}$

Toxic combination of vested interests and good intentions ${ }^{25}$

Supply-sensitive care ${ }^{17}$

Industry interest in expanding markets, including financial ties to those setting diagnostic thresholds ${ }^{3-32}$

Professionals

Fear of litigation or defensive medicine $e^{3-19}{ }_{-}^{23} 2{ }_{-}{ }_{-}^{30-41}$

Fear of missing disease or decision regret ${ }^{3-29}$

Tackle and reform drivers of litigation and defensive medicine ${ }^{3-2}$

Critical thinking and comfort with uncertainty (eg, with a period of active
surveillance, watchful waiting) $)^{12-35}$

Flaws in training:

Hard to do nothing rather than something ${ }^{9-23}$

Educational curricula and information materials on overdiagnosis and

Overemphasis on diagnosis

20

Problem based learning approach leads to shotgun approach to diagnosis, rewards unusual or rare diagnoses, and contributes to overtesting and overdiagnosis, with an emphasis on avoiding omission errors $^{4-4}$

Poor knowledge of patient preference and shared decision making ${ }^{23}$ Improper weighting of absolute $v$ relative risk ${ }^{8-44}$

Lack of professional self confidence or knowledge of harms $\mathrm{s}^{8-30}$ NA

Interventions to reduce overuse and overdiagnosis:

Multicomponent interventions tackling patient and provider roles (eg, clinician education with decision support or feedback) to reduce overuse and low value care ${ }^{10}$

Using minimally invasive techniques to eventually replace surgery for low risk tumours (eg, low staged lung tumours) ) $^{39}$

Clinical decision support and performance feedback ${ }^{1013}$

Compare practices with peers (eg, prescribing, referrals, tests) ${ }^{13}$

Deprescribing $^{44}$

Overusing or over-reliance on tests ${ }^{913}$ :

Reduce over-reliance on tests

Using tests to close consultation ${ }^{13}$

Chasing incidental findings and test outcomes of no or doubtful significance $^{9}$

Chart overload syndrome (order test to avoid reading thick chart) ${ }^{9}$

Specialists ordering tests before clinical appointment assessments ${ }^{9}$

Specialists suggesting unnecessary tests to primary provider, especially during short consultation or in corridor ${ }^{9}$

Incomplete information of test ordering requests ${ }^{9}$
Anticipate consequences of discovering abnormalities ${ }^{11}$

More time with patient to order less tests ${ }^{30} 46$

Tools to promote discussion with patients on why test might not be needed $^{13}$

Don't use diagnostic labels unless needed ${ }^{33}$
Consider stepped or slower diagnosis ${ }^{1733}$ 


\section{Table 1 (continued)}

\section{Possible drivers}

\section{Potential solutions}

Radiologists suggesting further imaging studies (self referral) when they don't have complete clinical information about patient ${ }^{9}$

Tests express "care" to patients and families, whereas watchful waiting deemed uncaring ${ }^{17}$

"Better to know" bias that may not be warranted ${ }^{8}$

Too much focus on surrogate markers ${ }^{20}$

Patients and public

Over-reliance on tests, including for reassurance ${ }^{8-28}$

NA

NA

Shared decision making ${ }^{13-21}{ }_{-}^{23-46}$

Lack of knowledge or confidence about limits and harms of medicine ${ }^{823}$

Information and education campaigns, with accessible evidence, on the benefits and harms of interventions, including screening, from unconflicted sources ${ }^{38}{ }_{-}^{10-46}$

Expectation that clinicians will "do something"9-2

Research and promote doing nothing, watchful waiting, or active

surveillance as legitimate options, where appropriate ${ }^{3-35}$

Some responses may map to more than one driver. NA=not applicable. *In most markets, costs are constrained by consumers' ability to pay; in health care, services are ordered by physicians, who are unaffected by cost, and received by patients, who often bear only a portion of the cost. 'Leading to test duplication, polypharmacy, and redundant opinions owing to one person seeking advice from several physicians. ${ }^{*}$ When patients are forced or indirectly encouraged to seek care through an inappropriate or inefficient avenue. For instance, all too frequently, the most expeditious route to urgent, but not emergent, care is through an emergency department. §Industry includes for-profit drug and device companies and commercial or corporate medicine providers. ๆWhere higher capacity in the form of hospital beds and imaging modalities drives medical use and inevitably uncovers patient abnormalities 


\section{Figures}

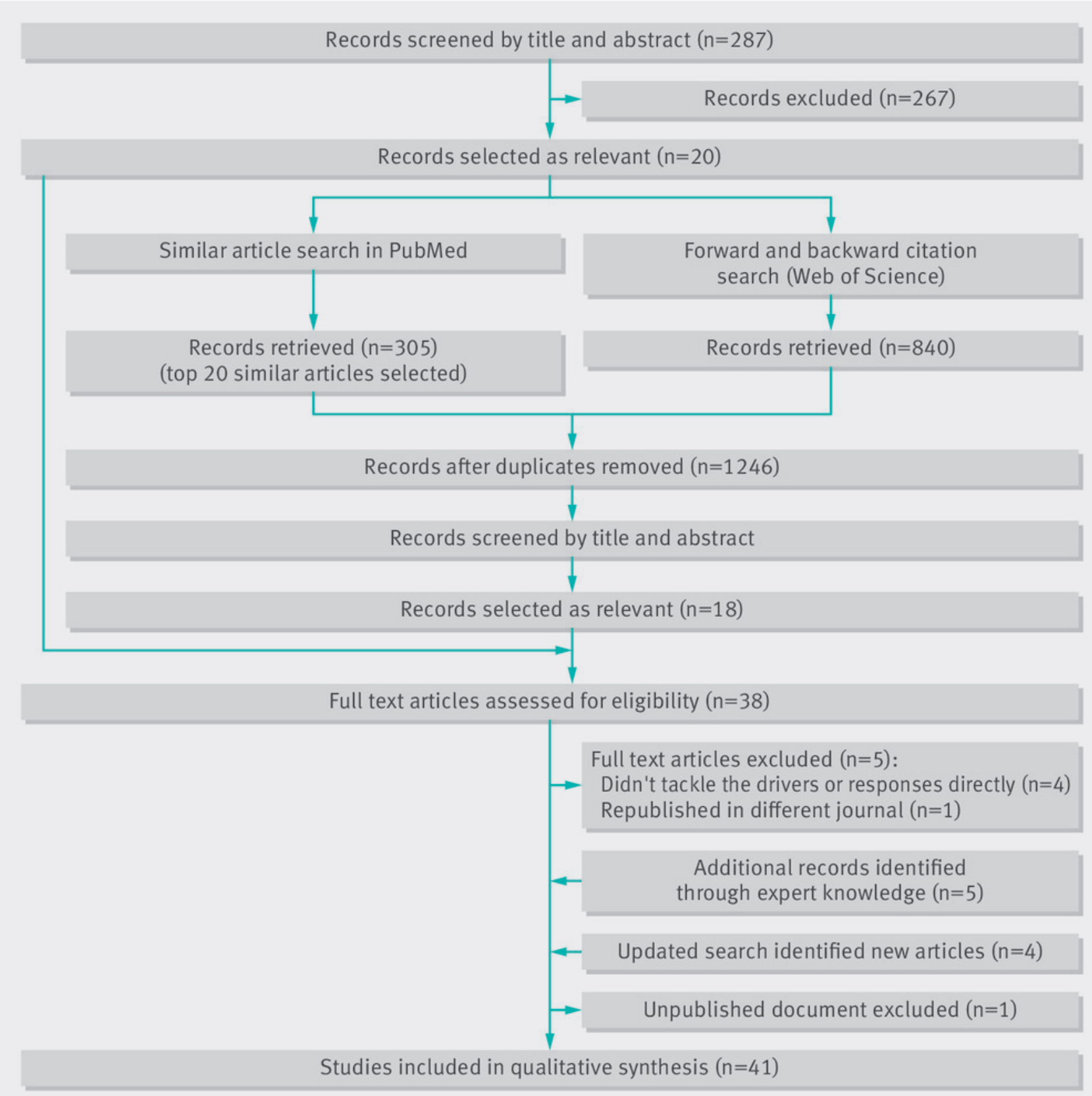

Fig 1 Flow diagram 


\section{- Possible drivers}

\section{Culture}

- Beliefs; for example, more = better

- Faith in early diagnosis

- Intolerance of uncertainty

- Biased media reporting

- Medicalisation

\section{Health system}

- Financial incentives

- Expanding disease definitions

- Quality measures

- Complexity of care

- Guidelines

- Screening

Industry and technology

- Industry promotion

- Diagnostic test sensitivity

- Medicine as a business

- Industry expands markets

\section{Professionals}

- Fear of litigation

- Fear of missing disease

- Flaws in training

- Lack of confidence or knowledge

- Over-reliance on tests

Patients and public

- Over-reliance on tests

- Lack of confidence or knowledge

- Expectation clinicians will "do something"

\section{$\checkmark$ Possible solutions}

Culture

$\checkmark$ Awareness/information campaigns $\checkmark$ Healthy scepticism about early diagnosis

$\checkmark$ Address uncertainty $\checkmark$ Improve media reporting

Culture

Health system $\checkmark$ Reform incentives from quantity to quality $\checkmark$ Reform disease definition $\checkmark$ Reform quality measures $\checkmark$ Reform guidelines $\checkmark$ Reform screening More research on OD and OU $\checkmark$ Multicomponent interventions

Industry and technology $\checkmark$ Better regulate promotion $\checkmark$ Better evaluation of tests $\checkmark$ Declare, reduce, exclude COIs $\checkmark$ Better evaluate disease definitions

Professionals

$\checkmark$ Reform litigation driver $\checkmark$ Comfort with uncertainty $\checkmark$ Educate and inform $\checkmark$ Interventions for providers $\checkmark$ Reduce test over reliance

Patients and public $\checkmark$ Shared decision making $\checkmark$ Education and information campaigns $\checkmark$ Promote "doing nothing"

Fig 2 Overdiagnosis and related overuse. Mapping possible drivers to potential solutions. $\mathrm{COI}=\mathrm{conflict}$ of interest; $\mathrm{OD}=$ overdiagnosis; $\mathrm{OU}=$ overuse. 